\title{
Attitudes and perceptions towards multiparametric magnetic resonance imaging of the prostate: A national survey among Italian urologists
}

\author{
Arnaldo Stanzione ${ }^{1}$, Massimiliano Creta ${ }^{2}$, Massimo Imbriaco ${ }^{1}$, Roberto La Rocca ${ }^{2}$, Marco Capece ${ }^{2}$, \\ Fabio Esposito ${ }^{2}$, Ciro Imbimbo ${ }^{2}$, Ferdinando Fusco ${ }^{3}$, Giuseppe Celentano ${ }^{2}$, Luigi Napolitano ${ }^{2}$, \\ Francesco Mangiapia ${ }^{2}$, Vincenzo Mirone ${ }^{2}$, Nicola Longo ${ }^{2}$ \\ ${ }^{1}$ Department of Advanced Biomedical Sciences, University of Naples "Federico II", Naples, Italy; \\ ${ }^{2}$ Department of Neurosciences, Reproductive Sciences and Odontostomatology, University of Naples "Federico II", Naples, Italy; \\ ${ }^{3}$ Department of Woman Child and of General and Specialist Surgery, University of Campania "Luigi Vanvitelli", Naples, Italy.
}

\begin{abstract}
Summary Objective: We aimed to assess the attitudes and perceptions towards multiparametric magnetic resonance imaging (mpMRI) of the prostate among Italian urologists.

Material and Methods: A national, web-based survey was performed. A questionnaire composed of 18 multiple choice questions was e-mailed to 941 currently active urologists, members of the Italian Society of Urology. Preserving anonymity, respondents' demographics were collected (e.g. geographic region, type of workplace, prostate procedures performed) as well as data concerning their attitudes and perceptions towards mpMRI (e.g. indications deemed appropriate, degree of confidence in mpMRI results). Data were expressed as raw numbers and percentages of survey answers.

Results: In total, 98 responses were received (participation rate $=10.4 \%$ ). Respondents mostly worked in urban areas (96\%) and primarily in hospital settings (89\%), while $48 \%$ of them worked in southern Italy. 97\% of respondents considered mpMRI useful to detect Prostate Cancer (PCa) in patients with prior negative biopsy, 64\% in biopsy-naïve patients and $60 \%$ for PCa pre-operatory staging. About half (42\%) of the participants declared that mpMRI results frequently lead them to change PCa management strategy. Standardization of mpMRI acquisition and reporting was partially unsatisfactory.

Reported waiting time for mpMRI scans was longer than 4 weeks for $51 \%$ of respondents. The major limitation of this survey includes the small number of participants.

Conclusions: Prostate mpMRI is used by Italian urologists mainly for detection and for pre-operative staging of PCa. Further improvements in terms of mpMRI availability and report standardization are required.
\end{abstract}

KEY WORDS: Multiparametric magnetic resonance imaging; Prostate cancer; Prostate imaging reporting and data system; Prostate biopsy; Survey.

Submitted 5 July 2020; Accepted 3 September 2020

\section{INTRODUCTION}

Multiparametric magnetic resonance imaging (mpMRI) of the prostate has emerged as a valuable tool for the detection of clinically significant prostate cancer (PCa) and the acceptance of this novel technology within the urology community has steadily increased (1). Current European Association of Urology (EAU) Guidelines strongly recommend prostate mpMRI before re-biopsy in patients with persistent suspicion of PCa despite a prior negative biopsy and before confirmatory biopsy in PCa patients on active surveillance (AS) (2). Further potential applications of prostate mpMRI include detection of PCa in biopsy naive patients, PCa staging prior to treatment and suspicion of local PCa recurrence $(1,2)$.

The technique has undergone progressive refinements over time regarding acquisition protocol, image interpretation and reporting $(3,4)$.

In 2012, the European Society of Urogenital Radiology established clinical guidelines for the acquisition, interpretation, and reporting of prostate mpMRI in order to allow an adequate level of standardization and consistency (5). These recommendations, popularly referred to as Prostate Imaging - Reporting and Data System (PI-RADS), were based on literature evidence and consensus expert opinion (5).

In 2014, the PI-RADS version 2 was officially launched (6). Currently, the standard mpMRI protocol combines morphological information derived from high spatial resolution T2-weighted images, and functional data concerning cell density (diffusion weighted imaging [DWI) with apparent diffusion coefficient [ADC) maps) and vascularization (dynamic contrast-enhanced imaging) (7). Despite the efforts to improve prostate mpMRI adoption and quality of reports, the acceptance and the standardization of the procedure in everyday clinical practice still suffer from many limitations. Indeed, mpMRI is not readily available at all institutions, and facilities' adherence to technical standards is variable and sometimes suboptimal (8).

Attitudes and perceptions toward prostate mpMRI vary across countries and, to date, these aspects have not been investigated in Italy (9-12). The goal of the present survey was to investigate patterns of attitudes and perceptions among practicing Italian urologists with regard to the use of mpMRI to manage PCa as well as mpMRI availability and reporting quality. 


\section{MATERIALS AND MEthodS}

\section{Questionnaire}

An online survey consisting of 18 multiple choice questions (formulated in Italian with the aim of increasing the response rate) was designed using the Google Form application included in the Google Drive office suite (Google LLC). The questionnaire was composed of two sections: a first one to assess respondents' demographics and a second one to evaluate their attitudes and perceptions (i.e. prescription attitudes; mpMRI availability; reporting quality; sequences considered useful in clinical practice; perceptions toward the usefulness of the procedure; impact of mpMRI results on the decision making process; management of PI-RADS 3 lesions; type of targeted prostate biopsy performed or recommended). In particular, questions about demographics included: years since completion of residency, type and location of medical practice, execution of prostate biopsies, and number of prostatectomies performed monthly. mpMRI availability was addressed by asking about average waiting time for the exam. Questions about attitudes toward mpMRI indications investigated the following potential settings: biopsy naive patients, patients with clinical suspicious $\mathrm{PCa}$ despite a previous negative biopsy, pre-treatment staging, suspicion of local recurrence, AS. Questions about reporting quality addressed: the description of the sequences used, the adoption of the PI-RADS version 2 scoring system, and the presence of a segmentation map with clear identification of the index lesion in the reports received. Questions about perceptions toward the clinical utility of prostate mpMRI were formulated to assess the perceived reliability of PI-RADS v2 in identifying clinically significant PCa, and the utility in the evaluation of local stage and recurrences. Some questions required a single answer while others gave the respondents the choice to select as many answers as they felt appropriate.

\section{Data collection}

Invitations to participate in this anonymous survey were emailed on 28 January 2019 to 941 current Italian Society of Urology (SIU) members who gave the approval to the use of their e-mail address. All survey participants were practicing urologists in Italy. For those who had not completed the survey, four follow-up reminder e-mail invitations were sent over the following 2 weeks. The survey was closed on 28 February 2019. All respondents had to fully complete the questionnaire before submission since all questions were flagged as mandatory. After submission, users could not review neither amend their answers. Both personal contact information and data collected were not accessible to third parties.

\section{Data analysis}

Data were expressed as raw numbers and percentages of survey answers. Chi square and Fisher's exact tests were used to assess variability in responses between demo-

Figure 1.
Table 1.

Survey demographics and mpMRI of the prostate availability.

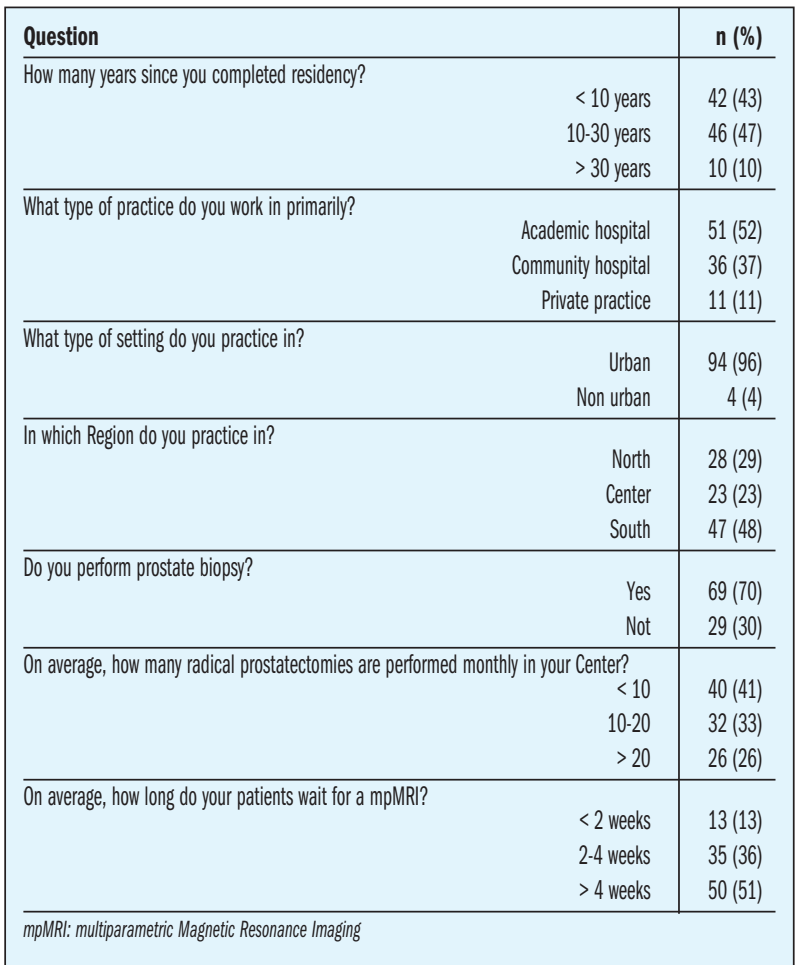

graphic groups and according to mpMRI availability. Statistical analyses were two-sided using a significance level of 0.05. All statistical analyses were performed with SPSS version 17.0 (SPSS, Inc., Chicago, IL) software.

\section{RESULTS}

A total of 98 responses were received from the survey within 30 days of the initial request (participation rate $=$ 10.4\%). Table 1 shows demographic data of the respondents and information about average waiting time for prostate mpMRI. Ninety percent of respondents $(n=88)$

Attitudes toward the prescription of mpMRI of the prostate in the various clinical settings.

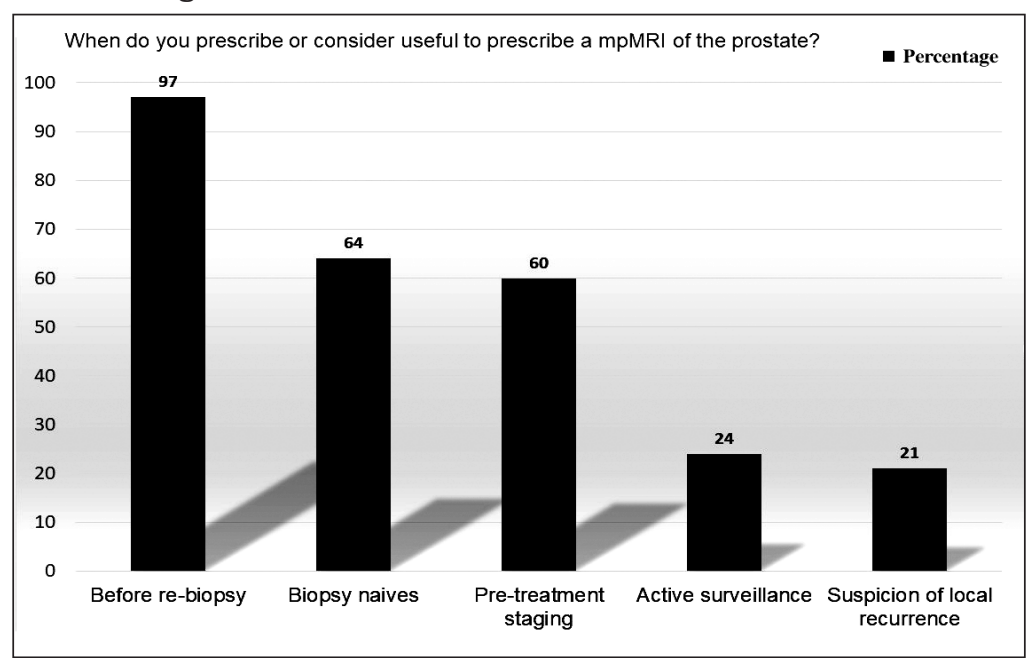


Table 2.

Urologists' attitudes and perceptions toward mpMRI of the prostate.

\begin{tabular}{|c|c|}
\hline Question & $n(\%)$ \\
\hline \multicolumn{2}{|l|}{ When do you prescribe or consider useful to prescribe a mpMRI of the prostate? } \\
\hline Biopsy naïve patients & $63(64)$ \\
\hline Before re-biopsy & $95(97)$ \\
\hline Pre-operatory staging & $59(60)$ \\
\hline Suspicion of local recurrence & $21(21)$ \\
\hline Active survellance & $24(24)$ \\
\hline \multicolumn{2}{|l|}{$\begin{array}{l}\text { Which of the following sequences do you consider useful } \\
\text { for your clinical practice? }\end{array}$} \\
\hline High resolution T2-weighted alone & $9(9)$ \\
\hline Axial DWI with ADC maps alone & $3(3)$ \\
\hline Axial perfusion alone & $3(3)$ \\
\hline High resolution T2-weighted + Axial DWI with ADC maps & $24(25)$ \\
\hline High resolution T2-weighted + Axial perfusion & $2(2)$ \\
\hline Axial DWI with ADC maps + Axial perfusion & $7(7)$ \\
\hline High resolution T2-weighted + Axial DWI with ADC maps + Axial perfusion & $39(40)$ \\
\hline High resolution T2-weighted + Axial DWI with ADC maps + Axial perfusion + Spectroscopy & $8(8)$ \\
\hline Axial DWI with ADC maps + Axial perfusion + Spectroscopy & $2(2)$ \\
\hline High resolution T2-weighted + Axial DWI with ADC maps + Spectroscopy & $1(1)$ \\
\hline \multicolumn{2}{|l|}{ How do you manage PI-RADS 3 lesions? } \\
\hline No further investigations & $1(1)$ \\
\hline Secondary interpretation in referral centers & $25(26)$ \\
\hline Targeted prostate biopsy as for PI-RADS 4 and 5 lesions & $25(26)$ \\
\hline Targeted prostate biopsy only in cases of high clinical suspicious & $20(19)$ \\
\hline Standard biopsy & $27(28)$ \\
\hline \multicolumn{2}{|l|}{ How often results from mpMRI change your strategy? } \\
\hline Almost always & $5(5)$ \\
\hline Often & $36(37)$ \\
\hline Sometimes & $52(53)$ \\
\hline Hardly ever & $5(5)$ \\
\hline \multicolumn{2}{|l|}{ Which technique of targeted prostate biopsy do you perform or advise? } \\
\hline Visual-cognitive & $46(47)$ \\
\hline Software-assisted registration MRI-TRUS & $49(50)$ \\
\hline MRl guidance & $3(3)$ \\
\hline \multicolumn{2}{|l|}{$\begin{array}{l}\text { In your clinical practice, how do you rate the reliability of PI-RADS v2 } \\
\text { in identifying clinically significant PCa? }\end{array}$} \\
\hline Highly reliable & $21(21)$ \\
\hline Reliable & $75(76)$ \\
\hline Unreliable & 2 (2) \\
\hline \multicolumn{2}{|l|}{$\begin{array}{l}\text { In your clinical practice, how do you rate the reliability of mpMRI } \\
\text { in local staging of } \mathrm{PCa} \text { ? }\end{array}$} \\
\hline Highly reliable & $24(24)$ \\
\hline Reliable & $69(70)$ \\
\hline Unreliable & $5(5)$ \\
\hline \multicolumn{2}{|l|}{$\begin{array}{l}\text { In your clinical practice, how do you rate the reliability of mpMRI } \\
\text { in the evaluation of local recurrence? }\end{array}$} \\
\hline Highly reliable & $11(11)$ \\
\hline Reliable & $68(69)$ \\
\hline Unreliable & $19(20)$ \\
\hline
\end{tabular}

ADC: Apparent Diffusion Coefficient; DWI: Diffusion Weighted Imaging; mpMRl: multiparametric Maǵnetic Resonance Imaging; PI-RADS: Prostate Imaging - Reporting and Data System.

completed residency less than 30 years before the survey. About half of them $(48 \%, \mathrm{n}=47)$ worked in Southern Italy, 96\% ( $\mathrm{n}=94)$ in urban areas, and 89\% (n $=87$ ) primarily in a hospital setting. Seventy percent of respondents $(n=69)$ declared to personally perform prostate biopsy and about half of them $(51 \%, \mathrm{n}=50)$ declared that their patients wait more than 4 weeks for a prostate mpMRI. Urologists' attitudes toward prostate mpMRI prescription in various clinical scenarios are reported in Table 2. The prevalence of indications for prostate mpMRI in the settings explored are shown in
Table 3.

Indications to mpMRI according to demographic features and $\mathrm{mpMRI}$ availability.

\begin{tabular}{|c|c|c|c|c|c|c|}
\hline & & $\begin{array}{c}\text { Biopsy } \\
\text { naive } \\
\text { patients } \\
(n=63)\end{array}$ & $\begin{array}{c}\text { Before } \\
\text { repeat } \\
\text { biopsy } \\
(n=95)\end{array}$ & $\begin{array}{c}\text { Pre- } \\
\text { operatory } \\
\text { staging } \\
(n=59)\end{array}$ & $\begin{array}{c}\text { Suspect } \\
\text { local } \\
\text { recurrence } \\
(n=21)\end{array}$ & $\begin{array}{l}\text { Patients } \\
\text { active } \\
\text { surveillance } \\
(n=24)\end{array}$ \\
\hline \multicolumn{7}{|c|}{ Years since completition } \\
\hline \multirow[t]{4}{*}{ of residency } & $<10(n=42)$ & $28(66.7 \%)$ & $41(97.6 \%)$ & $26(61.9 \%)$ & $8(19.0 \%)$ & $11(26.2 \%)$ \\
\hline & $10-30(n=46)$ & $27(58.7 \%)$ & $44(95.7 \%)$ & $25(54.3 \%)$ & $10(21.7 \%)$ & $12(26.1 \%)$ \\
\hline & $>30(n=10)$ & $8(80.0 \%)$ & $10(100 \%)$ & $8(80.0 \%)$ & $3(30.0 \%)$ & $1(10.0 \%)$ \\
\hline & $p$ & 0.419 & 1.000 & 0.316 & 0.682 & 0.657 \\
\hline \multicolumn{7}{|l|}{ Setting of practice } \\
\hline Academ & hospital $(n=51)$ & $33(64.7 \%)$ & $50(98.0 \%)$ & $30(58.8 \%)$ & $14(27.5 \%)$ & $14(27.5 \%)$ \\
\hline Commun & hospital $(n=36)$ & $23(63.9 \%)$ & $36(100 \%)$ & $22(61.1 \%)$ & $6(16.7 \%)$ & $8(22.2 \%)$ \\
\hline Priva & practice $(n=11)$ & $7(63.6 \%)$ & $9(81.8 \%)$ & $7(63.6 \%)$ & $1(9.1 \%)$ & $2(18.2 \%)$ \\
\hline & $p$ & 0.999 & 0.032 & 0.999 & 0.333 & 0.792 \\
\hline \multicolumn{7}{|l|}{ Region } \\
\hline & North $(n=28)$ & $23(82.1 \%)$ & $26(92.9 \%)$ & $18(64.3 \%)$ & $2(7.1 \%)$ & $8(28.6 \%)$ \\
\hline & Center $(n=23)$ & $10(43.5 \%)$ & $23(100 \%)$ & $17(73.9 \%)$ & $5(21.7 \%)$ & $4(17.4 \%)$ \\
\hline & South $(n=47)$ & $30(63.8 \%)$ & $46(97.9 \%)$ & $24(51.1 \%)$ & $14(29.8 \%)$ & $12(25.5 \%)$ \\
\hline & $p$ & 0.016 & 0.438 & 0.162 & 0.060 & 0.652 \\
\hline \multicolumn{7}{|c|}{ Do you perform biopsy? } \\
\hline & Yes $(n=69)$ & $42(60.9 \%)$ & $68(98.6 \%)$ & $40(58.0 \%)$ & $15(21.7 \%)$ & $17(24.6 \%)$ \\
\hline & No $(n=29)$ & $21(72.4 \%)$ & $27(93.1 \%)$ & $19(65.5 \%)$ & $6(20.7 \%)$ & $7(24.1 \%)$ \\
\hline & $p$ & 0.276 & 0.208 & 0.486 & 0.907 & 0.958 \\
\hline \multicolumn{7}{|c|}{ Radical prostatectyomies } \\
\hline performed in the & $<10(n=40)$ & $19(47.5 \%)$ & $39(97.5 \%)$ & $24(60.0 \%)$ & $11(27.5 \%)$ & $8(20.0 \%)$ \\
\hline working center & $10-20(n=32)$ & $25(78.1 \%)$ & $30(93.8 \%)$ & $19(59.4 \%)$ & $6(18.8 \%)$ & $10(31.3 \%)$ \\
\hline \multirow[t]{2}{*}{ per month } & $>20(n=26)$ & $19(73.1 \%)$ & $26(100 \%)$ & $16(61.5 \%)$ & $4(15.4 \%)$ & $6(23.1 \%)$ \\
\hline & $p$ & 0.014 & 0.483 & 0.985 & 0.495 & 0.533 \\
\hline \multicolumn{7}{|c|}{ Waiting time for mpMRI } \\
\hline & $<2$ weeks $(n=13)$ & $8(61.5 \%)$ & $12(92.3 \%)$ & $5(38.5 \%)$ & $4(30.8 \%)$ & $6(46.2 \%)$ \\
\hline & -4 weeks $(n=35)$ & $23(65.7 \%)$ & $33(94.3 \%)$ & $24(68.6 \%)$ & $7(20.0 \%)$ & $6(17.1 \%)$ \\
\hline & 4 weeks $(n=50)$ & $32(64.0 \%)$ & $50(100 \%)$ & $30(60.0 \%)$ & $10(20.0 \%)$ & $12(24.0 \%)$ \\
\hline & $p$ & 0.962 & 0.139 & 0.166 & 0.463 & 0.114 \\
\hline
\end{tabular}

Figure 1. In particular, the following values were found: suspicious $\mathrm{PCa}$ in patients with prior negative biopsy (97\%, $\mathrm{n}=95)$, suspicious PCa in biopsy naive patients $(64 \%, \mathrm{n}=63)$, pre-operatory staging $(60 \%, \mathrm{n}=59)$, active surveillance $(24 \%, n=24)$, suspicious local recurrence $(21 \%, n=21)$. Geographic region and number of radical prostatectomies performed per month were significant factors influencing the prescription of prostate mpMRI in biopsy-naive patients while setting of practice was the only factor influencing the prescription of prostate mpMRI in the re-biopsy setting (Table 3).

Results from questions investigating the quality of reports received are showed in Table 4. Most respondents $(81 \%, \mathrm{n}=79)$ declared to receive reports including a list of the sequences acquired and evaluated. The percentages of respondents declaring to receive often or almost always a report interpreted using PI-RADS v2 guidelines and including a prostate segmentation map with the index lesions highlighted were $88 \%(n=86)$ and $78 \%(n=76)$, respectively.

mpMRI sequences judged as useful are showed in Table 2. Forty percent of respondents $(n=39)$ judged useful the combination of high resolution T2-weighted, axial DWI with ADC maps, and axial perfusion sequences. The percentages of respondents judging reliable or highly reliable mpMRI in identifying clinically significant $\mathrm{PCa}$, in local staging and in evaluating of local recur- 
Table 4.

mpMRI data reporting.

\begin{tabular}{|c|c|}
\hline Question & n (\%) \\
\hline \multicolumn{2}{|l|}{ Do mpMRI reports you receive include a list of MRI sequences acquired and evaluated? } \\
\hline Yes & $79(81)$ \\
\hline Not & $19(19)$ \\
\hline \multicolumn{2}{|l|}{$\begin{array}{l}\text { How often the mpMRI reports you receive are interpreted (and lesions scored) using } \\
\text { the PI-RADS v2 guidelines? }\end{array}$} \\
\hline Almost always & $62(63)$ \\
\hline Often & $24(25)$ \\
\hline Sometimes & $11(11)$ \\
\hline Hardly ever & $1(1)$ \\
\hline \multicolumn{2}{|l|}{$\begin{array}{l}\text { How often the mpMRI reports you receive include a prostate segmentation map } \\
\text { with the index lesions highlighted? }\end{array}$} \\
\hline Almost always & $45(46)$ \\
\hline Often & $31(32)$ \\
\hline Sometimes & $14(14)$ \\
\hline Hardly ever & $8(8)$ \\
\hline
\end{tabular}

rences were 97\%, 94\% and 79\%, respectively. Overall, $42 \%$ of them declared to change often or almost always PCa management strategy based on mpMRI results.

In total, only $26 \%$ of respondents declared to prescribe targeted biopsy in cases of PI-RADS 3 lesions as for PIRADS 4 and 5 lesions. The percentages of respondents declaring to perform or advise targeted prostate biopsy with software assisted registration MRI-ultrasound, visu-

Table 5.

Targeted biopsy modality of choice.

\begin{tabular}{|c|c|c|c|}
\hline & $\begin{array}{l}\text { Visual-cognitive } \\
\quad(n=46)\end{array}$ & $\begin{array}{l}\text { Software-assisted } \\
\text { registration MRI-TRUS } \\
(n=49)\end{array}$ & $\begin{array}{l}\text { MRI guidance } \\
\qquad(n=3)\end{array}$ \\
\hline \multicolumn{4}{|l|}{ Years since completition } \\
\hline of residency $\quad<10(n=42)$ & $19(45.2 \%)$ & $21(50.0 \%)$ & $2(4.7 \%)$ \\
\hline $10-30(n=46)$ & $24(52.1 \%)$ & $21(45.6 \%)$ & $1(2.1 \%)$ \\
\hline$>30(n=10)$ & $3(30.0 \%)$ & $7(70.0 \%)$ & $0(0 \%)$ \\
\hline$p$ & 0.429 & 0.398 & 0.714 \\
\hline \multicolumn{4}{|l|}{ Setting of practice } \\
\hline Academic hospital $(n=51)$ & $28(54.9 \%)$ & $23(45.0 \%)$ & $0(0 \%)$ \\
\hline Community hospital $(n=36)$ & $13(36.1 \%)$ & $20(55.5 \%)$ & $3(8.3 \%)$ \\
\hline Private practice $(n=11)$ & $5(45.4 \%)$ & $6(54.5 \%)$ & $0(0 \%)$ \\
\hline$p$ & 0.225 & 0.292 & 0.125 \\
\hline \multicolumn{4}{|l|}{$\overline{R e g i o n}$} \\
\hline North $(n=28)$ & $7(25.0 \%)$ & $19(67.8 \%)$ & $2(7.1 \%)$ \\
\hline Center $(n=23)$ & $13(56.5 \%)$ & $10(43.4 \%)$ & $0(0 \%)$ \\
\hline South $(n=47)$ & $26(55.3 \%)$ & $20(42.5 \%)$ & $1(2.1 \%)$ \\
\hline$p$ & 0.024 & 0.088 & 0.436 \\
\hline \multicolumn{4}{|l|}{ Do you perform biopsy? } \\
\hline Yes $(n=69)$ & $33(47.8 \%)$ & $33(47.8 \%)$ & $3(4.3 \%)$ \\
\hline No $(n=29)$ & $13(44.8 \%)$ & $16(55.1 \%)$ & $0(\%)$ \\
\hline$p$ & 0.786 & 0.506 & 0.552 \\
\hline \multicolumn{4}{|l|}{ RP performed in the working } \\
\hline $10-20(n=32)$ & $18(56.2 \%)$ & $13(40.6 \%)$ & $1(3.1 \%)$ \\
\hline$>20(n=26)$ & $6(23.0 \%)$ & $18(69.2 \%)$ & $2(7.6 \%)$ \\
\hline$p$ & 0.017 & 0.071 & 0.183 \\
\hline \multicolumn{4}{|l|}{ Waiting time for mpMRI } \\
\hline$<2$ weeks $(n=13)$ & $6(46.1 \%)$ & $7(53.8 \%)$ & $0(0 \%)$ \\
\hline $2-4$ weeks $(n=35)$ & $18(51.4 \%)$ & $17(48.5 \%)$ & $0(0 \%)$ \\
\hline$>4$ weeks $(n=50)$ & $22(44 \%)$ & $25(50.0 \%)$ & $3(6.0 \%)$ \\
\hline$p$ & 0.818 & 0.999 & 0.372 \\
\hline
\end{tabular}

al cognitive, and MRI guidance techniques were 50\%, $47 \%$, and 3\%, respectively. Geographic region and number of radical prostatectomies performed per month were significant factors influencing the adoption of visual cognitive technique (Table 5).

\section{Discussion}

The adoption of prostate mpMRI to detect and characterize prostate lesions and to tailor the management of PCa patients has evolved over the last 10 years $(11,13$ 15). Currently, however, the availability of this technology, the quality of reporting, and urologists' perceptions and attitudes toward significantly varies across countries and some authors have stressed the need to integrate prostate mpMRI teaching courses into the training of urologists (16). To the best of our knowledge, we performed the first survey examining prostate mpMRI availability, quality of reports as well as urologists' attitudes and perceptions toward this diagnostic modality in Italy. The response rate elicited was within the range of surveys on this topic $(10,11,17)$. Respondents seemed to look favorably upon use of prostate mpMRI, as 100\% of them declared to prescribe or consider useful to prescribe it in at least one setting. By comparison, the survey published by Muthigi and coworkers in 2017 found only $85.7 \%$ of urologists declaring to use prostate mpMRI in their practice (9).

The top settings for the use of prostate mpMRI was in patients with persistent $\mathrm{PCa}$ suspicion despite a prior negative biopsy (97\%), followed by PCa suspicion in biopsy naive patients (64\%) and pre-operatory staging (60\%). Accordingly, most of respondents had a positive perception toward the ability of mpMRI to identify clinically significant PCa and to provide an adequate local staging. The high percentage of respondents declaring to prescribe mpMRI in patients with persistent PCa suspicion despite a prior negative biopsy confirms the results from previous surveys from other counties $(9,11)$. Indeed, $89.5 \%$ of respondents in the survey by Muthigi and coworkers declared to prescribe prostate mpMRI in this clinical scenario (9). To date, there is strong evidence demonstrating that mpMRI-guided biopsy increases the detection rate for clinically significant PCa when compared to standard biopsies and current EAU Guidelines strongly recommend mpMRI prior to repeat biopsy (2). Interestingly, we found that urologists working in private practice setting were less prone to prescribe mpMRI before repeat biopsy.

The percentage of respondents prescribing mpMRI on biopsy naïve patients was higher if compared with published evidence. Indeed, previous surveys reported percentages of utilization of prostate mpMRI in this setting ranging from $36.8 \%$ to $53 \%(9,11)$. Evidences about prostate mpMRI in this setting are contradictory and current Guidelines provide only weak recommendations for the adoption of mpMRI in patients undergoing their first biopsy (2). However, results from the recent randomized multicenter PRECISION trial involving 500 biopsy-naïve patients with suspected PCa support the role of pre-biopsy prostate mpMRI and recommendations may change in the future (18). mpMRI is considered as the imaging modality of choice for local staging (19). Sixty percent of 
respondents in the present survey declared to prescribe mpMRI for pre-operatory staging. This finding is in line with published evidences as previous surveys from other countries reported percentages of utilization of mPMRI in this setting ranging from $38 \%$ to $85 \%(9,11,17)$.

Only a small percentage of respondents declared to prescribe prostate mpMRI in the clinical setting of AS (24\%) and suspect local recurrence (21\%). mpMRI has emerged as a valuable diagnostic modality in both patient selection and monitoring for men who undergoing AS for PCa and its use in this clinical scenario is gradually increasing (20-22). Current EAU Guidelines strongly recommend prostate mpMRI in men on AS before confirmatory prostate biopsy if not done before the first biopsy (2). However, multiple barriers have been reported to counteract the widespread use of mpMRI for AS including quality, cost and access to care (20). Accordingly, receipt of mpMRI among PCa patients on AS significantly vary across demographic, geographic, and socioeconomic strata (21). The percentage of respondents prescribing mpMRI in AS setting we reported is lower if compared to data obtained from published surveys. Indeed, $85 \%$ of French urologists and $66 \%$ of urologists working in the United States declared to utilize mpMRI in this setting $(11,17)$. Moreover, although published surveys involving urologists working France and in the United States demonstrated that the percentage of urologists prescribing prostate mpMRI in patients enrolled in AS was significantly higher among those working in academic hospitals, we failed to confirm this finding (11). Similar to AS setting, the percentage of urologists involved in the present survey that declared to prescribe mpMRI in patients with suspicious of local recurrence was lower when compared to those from other surveys. Indeed, $51 \%$ of French urologists declared to prescribe mpMRI with the intent to detect local recurrence following radical treatments (11).

Urologists prescribing prostate mpMRI need to be confident regarding the report they receive, as both their decision-making process and the quality of targeted prostate biopsy they perform mainly depends on the count, location, and radiographic stage of lesions identified by the radiologist $(16,23)$. Interestingly, about $22 \%$ of respondents in the present survey declared to receive reports that sometimes or hardly ever include a prostate segmentation map with the index lesions highlighted, 19\% declared to receive reports that do not include a list of MRI sequences acquired and evaluated, and 12\% declared to receive reports that sometimes or hardly ever are interpreted using the PI-RADS v2 guidelines. Taken together, these results underline the need to further improve in our Country the process of standardization of prostate mpMRI reporting.

We collected evidences about urologists' point of view concerning mpMRI acquisition protocol and relevance of included sequences. The answers collected seem to indicate that the urologists might not be completely aware of the dominant sequence structure of the PI-RADS v2 guidelines. Indeed, fewer than half responders (40\%) correctly identified the currently recommended protocol suggesting that the major revision of the original PIRADS has not been universally embraced among urolo- gists. Furthermore, it should also be noted that a relatively high percentage (25\%) of urologists indicated that a protocol without the perfusion sequence could be considered adequate for their patients. This is somewhat in line with a current trend advocating for the need of shorter, cheaper and less invasive MRI protocols and reinforces the ongoing debate about the role of perfusion sequences in prostate imaging (24-26).

The management of undetermined, PI-RADS 3 lesions has represented a controversial issue for many years. Accordingly, the attitude of respondents is heterogeneous with only $26 \%$ of them recommending targeted biopsies as for PI-RADS 4 and 5 lesions and 20\% performing targeted biopsies only in cases of high clinical suspicious. More recent EAU Guidelines, updated in March 2019, consider prostate lesions with a PI-RADS score $\geq 3$ as positive and strongly recommend performing targeted plus systematic biopsy in biopsy naive patients and targeted biopsy only in patients with prior negative biopsy (2).

The modality with which prostate lesions identified by mpMRI are targeted at biopsy vary considerably. Visualcognitive and software-assisted registration MRITransrectal ultrasound guidance were the most frequently adopted guidance modalities by respondents in the present survey with MRI-guidance being adopted by only $3 \%$ of interviewed. Of note, the adoption of visualcognitive guidance was significantly lower in Northern Italy and in centers where more than 20 radical prostatectomies are performed monthly. Of note, the modality of lesion targeting during prostate biopsy represents a controversial and evolving issue (27-29). Beyond technical aspects, tumor multifocality is frequently involved in the discrepancies between findings from mp-MRI, prostate biopsy, and surgical specimens (29). Although MRI-guidance may represent a promising technique of biopsy guidance, it is still considered a time-consuming and expensive procedure and further investigations are needed to identify the ideal candidates (30).

The major limitation of this survey includes the small number of participants. However, the response rate is within published ranges. Secondly, respondents are not fully representative of the overall community of Italian urologists as those working in private practice setting and rural areas are poorly represented. Moreover, like any survey, participant responses were limited to the available choices.

\section{Conclusions}

This survey shows that prostate mPMRI is routinely used by urologists in Italy mainly before biopsy and for preoperative staging purposes. mpMRI availability and report standardization are suboptimal.

\section{REFERENCES}

1. Cuocolo R, Stanzione A, Ponsiglione A, et al. Clinically significant prostate cancer detection on MRI: A radiomic shape features study. Eur J Radiol. 2019; 116:144-149.

2. Mottet N, van den Bergh R.C.N, Briers E, et al. EAU - EANM ESTRO - ESUR - SIOG Guidelines on Prostate Cancer, 2019. 
3. Weinreb JC, Barentsz JO, Choyke PL, et al. PI-RADS Prostate Imaging - Reporting and Data System: 2015, Version 2. Eur Urol. 2016; 69:16-40.

4. Manfredi M, Mele F, Garrou D, et al. Multiparametric prostate MRI: technical conduct, standardized report and clinical use. Minerva Urol Nefrol. 2018; 70:9-21.

5. Barrett T, Turkbey B, Choyke PL. PI-RADS version 2: what you need to know. Clin Radiol. 2015; 70:1165-76.

6. Turkbey B, Rosenkrantz AB, Haider MA, et al. Prostate Imaging Reporting and Data System Version 2.1: 2019 Update of Prostate Imaging Reporting and Data System Version 2. Eur Urol. 2019; 76:340-351.

7. Tewes S, Mokov N, Hartung D, et al. Standardized reporting of prostate MRI: comparison of the Prostate Imaging Reporting and Data System (PI-RADS) Version 1 and Version 2. PLoS One. 2016; 11:e0162879.

8. Esses SJ, Taneja SS, Rosenkrantz AB. Imaging facilities' adherence to PI-RADS $v 2$ minimum technical standards for the performance of prostate MRI. Acad Radiol. 2018; 25:188-195.

9. Muthigi A, Sidana A, George AK, et al. Current beliefs and practice patterns among urologists regarding prostate magnetic resonance imaging and magnetic resonance-targeted biopsy. Urol Oncol. 2017; 35:32.e1-32.e7.

10. Manley BJ, Brockman JA, Raup VT, et al. Prostate MRI: a national survey of Urologist's attitudes and perceptions. Int Braz J Urol. 2016; 42:464-71.

11. Renard-Penna R, Rouvière O, Puech P, et al. Current practice and access to prostate MR imaging in France. Diagn Interv Imaging. 2016; 97:1125-1129.

12. Bukavina L, Tilburt JC, Konety B, et al. Perceptions of prostate MRI and fusion biopsy of radiation oncologists and urologists for patients diagnosed with prostate cancer: results from a national survey. Eur Urol Focus. 2020; 6:273-279.

13. Tu X, Lin T, Cai D, et al. The optimal core number and site for MRI-targeted biopsy of prostate? A systematic review and pooled analysis. Minerva Urol Nefrol. 2020; 72:144-151.

14. De Luca S, Fiori C, Bollito E, et al. Risk of Gleason Score $3+4$ $=7$ prostate cancer upgrading at radical prostatectomy is significantly reduced by target biopsy compared to standard biopsy technique. Minerva Urol Nefrol. 2020; 72:360-368.

15. Russo F, Manfredi M, Panebianco V, et al. Radiological Wheeler staging system: a retrospective cohort analysis to improve the local staging of prostate cancer with multiparametric MRI. Minerva Urol Nefrol. 2019; 71:264-272.

16. Kasivisvanathan V, Ambrosi A, Giganti F, et al. A dedicated prostate MRI teaching course improves the ability of the urologist to interpret clinically significant prostate cancer on multiparametric MRI. Eur Urol. 2019; 75:203-204.

17. Tooker GM, Truong H, Pinto PA, Siddiqui MM. National survey of patterns employing targeted MRI/US guided prostate biopsy in the diagnosis and staging of prostate cancer. Curr Urol. 2019; 12:97-103.

18. Kasivisvanathan V, Rannikko AS, Borghi M, et al. MRI-targeted or standard biopsy for prostate-cancer diagnosis. N Engl J Med. 2018; 378:1767-1777.

19. Caglic I, Kovac V, Barrett T. Multiparametric MRI - local staging of prostate cancer and beyond. Radiol Oncol. 2019; 53:159-170.

20. Glass AS, Dall' Era MA. Use of multiparametric magnetic resonance imaging in prostate cancer active surveillance. BJU Int. 2019; 124:730-737.
21. Fam MM, Yabes JG, Macleod LC, et al. Increasing utilization of multiparametric magnetic resonance imaging in prostate cancer active surveillance. Urology. 2019; 130:99-105.

22. Höffkes F, Arthanareeswaran VK, Stolzenburg JU, Ganzer R. Rate of misclassification in patients undergoing radical prostatectomy but fulfilling active surveillance criteria according to the European Association of Urology guidelines on prostate cancer: a high-volume center experience. Minerva Urol Nefrol. 2018; 70:588-593.

23. Puech P, Randazzo M, Ouzzane A, et al. How are we going to train a generation of radiologists (and urologists) to read prostate MRI? Curr Opin Urol. 2015; 25:522-35.

24. Stanzione A, Cuocolo R, Cocozza S, et al. Detection of extraprostatic extension of cancer on biparametric MRI combining texture analysis and machine learning: preliminary results. Acad Radiol. 2019; 26:1338-1344.

25. Cuocolo R, Stanzione A, Rusconi G, et al. PSA-density does not improve bi-parametric prostate MR detection of prostate cancer in a biopsy naïve patient population. Eur J Radiol. 2018; 104:64-70.

26. Salvaggio $G$, Calamia M, Purpura P, et al. Role of apparent diffusion coefficient values in prostate diseases characterization on diffusion-weighted magnetic resonance imaging. Minerva Urol Nefrol. 2019; 71:154-160.

27. Pepe P, Pepe L, Panella P, Pennisi M. Can multiparametric ultrasound improve cognitive MRI/TRUS fusion prostate biopsy. Arch Ital Urol Androl. 2020; 92:89-92

28. D'Agostino D, Mineo Bianchi F, Romagnoli D, et al. MRI/TRUS FUSION guided biopsy as first approach in ambulatory setting: Feasibility and performance of a new fusion device. Arch Ital Urol Androl. 2020; 91:211-217.

29. Lourenço M, Pissarra P, E Brito DV, et al. Lesion location agreement between prostatic multiparametric magnetic resonance, cognitive fusion biopsy and radical prostatectomy piece. Arch Ital Urol Androl. 2020; 91:218-223.

30. D'Agostino D, Mineo Bianchi F, Romagnoli D, et al. Comparison between "In-bore" MRI guided prostate biopsy and standard ultrasound guided biopsy in the patient with suspicious prostate cancer: Preliminary results. Arch Ital Urol Androl. 2019; 91:87-92.

\section{Correspondence}

Arnaldo Stanzione, MD - arnaldostanzione@yahoo.it

Massimo Imbriaco, MD - mimbriaco@hotmail.it

Department of Advanced Biomedical Sciences, University of Naples "Federico II", Via Pansini 5, 80131 Naples (Italy)

Massimiliano Creta, MD (Corresponding Author) - max.creta@gmail.com

Roberto La Rocca, MD - robertolarocca87@gmail.com

Marco Capece, MD - drmarcocapece@gmail.com

Fabio Esposito, MD - fabioesposito025@gmail.com

Ciro Imbimbo, MD - ciro.imbimbo@unina.it

Giuseppe Celentano, MD - dr.giuseppecelentano@gmail.com

Luigi Napolitano, MD - nluigi89@libero.it

Francesco Mangiapia, MP - mangiapippo@libero.it

Vincenzo Mirone, MD - mirone@unina.it

Nicola Longo, MD - nicolalongo20@yahoo.it

Department of Neurosciences, Reproductive Sciences and Odontostomatology, University of Naples "Federico II" - Via Pansini 5, 80131 Naples (Italy)

Ferdinando Fusco, MD - ferdinando-fusco@libero.it

Department of Woman Child and of General and Specialist Surgery,

University of Campania "Luigi Vanvitelli" - 80131 Naples (Italy) 